\title{
ELETROQUÍMICA COMPUTACIONAL - PRINCÍPIOS E DIRETRIZES PARA UTILIZAÇÃO
}

\author{
Mauro Chierici Lopes* \\ Departamento de Química, Universidade Estadual do Centro-Oeste, 85040-080 Guarapuava - PR, Brasil
}

Recebido em 12/3/13; aceito em 14/6/13; publicado na web em 9/8/13

\begin{abstract}
COMPUTATIONAL ELECTROCHEMISTRY - PRINCIPLES AND GUIDELINES FOR USE. In this paper, a detailed guide for the application of computational electrochemistry is presented. The basic framework of the electrochemical models and their computational solutions are described. We highlighted that the availability of commercial software allows application of the technique by experimentalists with minimal mathematical and computational expertise. The most used packages are indicated. Simulations of typical examples are presented and some references cited to illustrate the wide applicability of computational electrochemistry.
\end{abstract}

Keywords: computational electrochemistry; dynamic electrochemistry; electrochemical modeling.

\section{INTRODUÇÃO}

A experimentação em eletroquímica está firmemente assentada sobre uma teoria fenomenológica que é constituída por um conjunto de equações diferenciais parciais descrevendo essencialmente o transporte das espécies em solução, a distribuição de potencial elétrico e a cinética de transferência de carga na interface eletrodo/eletrólito. A solução destas equações fornece relações teóricas entre as grandezas de interesse (corrente, potencial, concentração, impedância, etc.) que possibilitam a análise quantitativa dos experimentos. Especialmente no caso de técnicas dinâmicas, isto é, quando o sinal aplicado varia com o tempo, a solução das equações diferenciais é difícil e, em última análise, envolve algum tipo de aproximação numérica. Dentre as diversas abordagens utilizadas, a de maior êxito e generalidade é a transformação das equações diferenciais em equações integrais pelo uso de transformada de Laplace e do teorema da convolução e a subsequente solução destas equações integrais por métodos numéricos. Esta abordagem, originalmente introduzida por Delahay ${ }^{1}$ e largamente estendida por Nicholson e Shain, ${ }^{2}$ permite obter relações funcionais entre corrente e potencial, que para experimentos de varredura linear, por exemplo, tem a forma:

$$
i=n F C_{o}^{*} \sqrt{\pi D_{o} \beta} \chi(\beta t)
$$

onde $\chi(\beta t)$ é uma função do potencial adimensional $\beta t$, obtida através da solução numérica da equação integral. Nesta expressão $\beta=\alpha n F / R T$, $\alpha$ é o coeficiente de transferência, $\mathrm{F}$ a constante de Faraday, $\mathrm{R}$ a constante universal dos gases, T a temperatura absoluta, $C_{O}^{*}$ a concentração da espécie eletroativa no seio da solução e $D_{O}$ o coeficiente de difusão da espécie eletroativa. Outros métodos, nos quais as equações integrais são resolvidas por expansão em série, foram igualmente aplicados com sucesso. ${ }^{3-5}$

Embora estas soluções semi-analíticas sejam importantes para uma descrição teórica geral dos fenômenos eletroquímicos, elas são obtidas mediante um conjunto de hipóteses simplificadoras que limitam a sua aplicabilidade em situações mais complexas. As aproximações mais comuns envolvem: simplificação na geometria do sistema assumindo geometria planar; simplificação no mecanismo cinético envolvido, desconsiderando adsorção, por exemplo; simplificação nos mecanismos de transporte, ignorando migração; simplificação na distribuição de potencial do sistema, ignorando resistências não

*e-mail: mauro@unicentro.br compensadas e correntes capacitivas. Existem numerosas situações de interesse prático para as quais as hipóteses simplificadoras acima mencionadas não são aceitáveis. Nestas condições, para obter algum resultado útil a partir da teoria fenomenológica, é necessário lançar mão de uma estratégia puramente numérica a partir da qual as equações diferenciais são solucionadas através de um método numérico apropriado sem nenhum tratamento analítico prévio. Embora algumas limitações inerentes aos métodos numéricos ainda existam em problemas mais complexos, esta abordagem amplia consideravelmente a gama de problemas que podem ser tratados.

O estudo de sistemas cada vez mais complexos associado ao aumento da capacidade de processamento dos computadores tem impulsionado este tipo de abordagem a partir da qual todas as técnicas dinâmicas e estacionárias podem ser tratadas sem a necessidade de simplificações nos modelos. Além disso, o desenvolvimento de modernos softwares tornou o processo de modelagem e simulação muito mais automático, não exigindo conhecimentos avançados de matemática e computação para sua aplicação. Esta área de investigação que tem recebido a denominação de eletroquímica computacional tem sido objeto de excelentes artigos de revisão. ${ }^{6-9}$

$\mathrm{O}$ rápido crescimento da eletroquímica computacional pode ser constatado com uma pesquisa por palavras-chave na base bibliográfica Scopus. A fração de artigos de eletroquímica que envolve modelagem computacional saltou de $1 \%$ para $5 \%$ em três décadas. Entretanto, na comunidade eletroquímica brasileira, este tipo de abordagem parece não ter ganhado a mesma aceitação. Enquanto a produção de artigos em eletroquímica de pesquisadores com filiação no Brasil nos últimos dez anos supera os $2 \%$ da produção mundial, em artigos relacionados à eletroquímica computacional a contribuição nacional é insignificante. ${ }^{10,11}$

Considerando esta lacuna, o presente trabalho se apresenta com um duplo propósito: mostrar as inúmeras possibilidades de aplicação da eletroquímica computacional e oferecer um guia para sua utilização.

O artigo está organizado da seguinte forma: a seção seguinte apresenta uma breve discussão sobre as ferramentas computacionais disponíveis para a modelagem de problemas de eletroquímica. Uma descrição geral das principais etapas do processo de modelagem e simulação de sistemas eletroquímicos é apresentada na seção "construindo um modelo". Considerações acerca da exatidão das soluções numéricas são apresentadas na seção "métodos numéricos". Na seção "exemplos de aplicação" são apresentados alguns exemplos de aplicações da eletroquímica computacional e na última seção algumas considerações finais. 


\section{FERRAMENTAS PARA A ELETROQUÍMICA COMPUTACIONAL}

Nas últimas três décadas, um esforço considerável de pesquisa tem sido feito para desenvolvimento e adaptação de métodos numéricos aos problemas eletroquímicos. ${ }^{12-16}$ Este intenso trabalho de pesquisa resultou no desenvolvimento de softwares especificamente dedicados à simulação eletroquímica (ES, do inglês "electrochemical software") que já se encontram comercialmente disponíveis: DIGISIM $^{\circledR},{ }^{17}$ DIGIELCH $^{\circledR 18}$ ou ELSIM ${ }^{\circledR} .{ }^{19}$ Nestes programas, os detalhes da formulação matemática do problema e sua solução computacional são completamente invisíveis para o usuário final, o que torna seu uso extremamente simples. Entretanto, os programas estão restritos a certo número de problemas pré-definidos, em geral, unidimensionais, o que torna impossível a simulação de situações não convencionais ou de novos problemas. Uma estratégia mais flexível é a utilização de pacotes comerciais de elementos finitos (FES, do inglês "finite element software") como COMSOL Multiphysics ${ }^{\circledR},{ }^{20}$ FlexPDE ${ }^{\circledR},{ }^{21}$ Ansys $^{\circledR}{ }^{22}$ São pacotes genéricos muito utilizados em problemas de engenharia para os quais praticamente não há limite do tipo de problema que pode ser tratado. Contudo, sua utilização é menos direta do que no primeiro caso, uma vez que o usuário deve configurar a geometria do problema, selecionar equações e condições de contorno a serem utilizadas e configurar alguns parâmetros relativos à solução numérica do problema. Outra desvantagem é o custo mais elevado das licenças.

\section{CONSTRUINDO UM MODELO}

Nesta seção enfocaremos a estrutura básica que se deve ter em mente para a definição completa de um modelo, tendo em vista a utilização dos softwares mencionados na seção anterior. Seguindo a discussão apresentada por John Alden, ${ }^{23}$ nosso ponto de partida serão as informações essenciais que definem um modelo eletroquímico: o mecanismo do processo eletroquímico que está sendo estudado, a geometria do eletrodo empregado neste estudo e a técnica experimental utilizada. A partir destas informações, são definidos os elementos constitutivos do modelo que são: o domínio, as equações e as condições de contorno.

\section{Definição do domínio}

Uma vez que os modelos matemáticos envolvem variáveis espaciais, uma descrição geométrica do sistema estudado é necessária. A região delimitada do espaço para a qual as equações diferenciais do modelo são válidas é denominada domínio do problema e em geral é representado pela letra $\Omega$. Os limites deste domínio são denominados de fronteiras e representados por $\delta \Omega$.

Nos ES, as geometrias são predefinidas a partir do tipo de eletrodo utilizado e o usuário apenas escolhe a opção apropriada. Elas são, em geral, limitadas aos casos unidimensionais e aos casos bidimensionais mais comuns. Nos FES uma geometria do problema deve ser definida utilizando ferramentas de desenho, o que confere extrema flexibilidade ao tipo de problema que pode ser estudado.

Considere, por exemplo, a modelagem de um sistema microfluídico constituído por um microcanal de largura $l$ e altura $h$ que está construído sobre um eletrodo de microbanda impresso de largura $w$. Um desenho esquemático do sistema é apresentado na Figura 1, mostrando os eletrodos impressos em escala aumentada para facilitar a visualização. Assumindo fluxo laminar e considerando que a largura do canal é muito maior que a largura do eletrodo, uma descrição bidimensional no plano perpendicular à superfície do eletrodo e paralelo à direção do fluxo é suficiente. Note que o retângulo tracejado e representado em destaque na Figura $1 \mathrm{~b}$ constitui o domínio utilizado para a simulação computacional deste sistema.

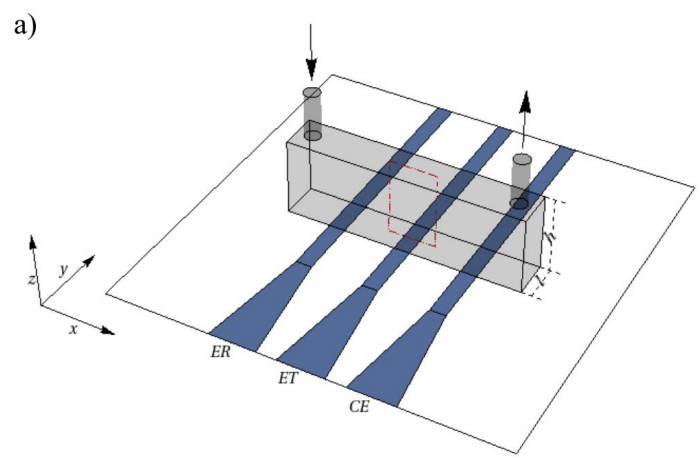

b)

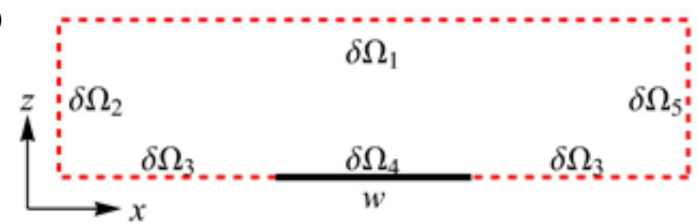

Figura 1. Definição do domínio para um sistema microfluídico com eletrodos de microbanda: a) Representação esquemática tridimensional do sistema indicando o plano correspondente ao domínio na região tracejada; $b$ ) Representação do domínio no plano $x-z$ onde h corresponde à altura do canal e $w$ corresponde à largura do eletrodo

\section{Definição das equações}

A maioria das situações experimentais em eletroquímica pode ser modelada pelo sistema formado pelas equações (2) e (3), que na literatura especializada recebe o nome de equações de NernstPlanck-Poisson (NPP).

$$
\begin{gathered}
\frac{\partial c_{i}}{\partial t}=\nabla \cdot\left(D_{i} \nabla c_{i}-u c_{i}+\mu_{i} \nabla \phi\right)+R\left(c_{i}, c_{j}\right) \\
\nabla \cdot(\varepsilon \nabla \phi)=-\rho
\end{gathered}
$$

A equação de Nernst-Planck (equação 2) é uma equação de conservação de massa que descreve o movimento das espécies químicas em solução. Ela expressa a variação da concentração $\left(c_{i}\right)$ da espécie i com o tempo em função da divergência dos fluxos por difusão $\left(D_{i} \nabla c_{i}\right.$, onde $D_{i}$ é o coeficiente de difusão da espécie i), convecção $\left(u c_{i}\right.$, onde $u$ indica a velocidade da solução) e migração $\left(\mu_{i} \nabla \phi\right.$, onde $\mu_{i}$ indica a mobilidade iônica da espécie i). Um termo reacional $\left(R\left(c_{i}, c_{j}\right)\right)$ foi adicionado para ter em conta reações químicas homogêneas entre as diferentes espécies. A equação de Poisson (equação 3) é uma equação clássica da eletrostática que permite calcular o potencial elétrico $(\phi)$ para uma dada distribuição de densidade de carga $(\rho)$ em um meio com permissividade elétrica $\varepsilon$. A aplicação destas equações aos principais experimentos eletroquímicos encontra-se bem documentada em diversos livros textos. ${ }^{24-26}$

Nesta seção consideraremos brevemente de que forma o mecanismo do processo eletroquímico e a técnica experimental utilizada definem as equações de Nernst-Planck-Poisson a serem utilizadas.

Evidentemente, o primeiro termo do lado direito da equação (2) deve ser escrito de acordo com os modos de transporte considerados para cada espécie. Assim, para o exemplo considerado na Figura 1, os termos relativos à difusão e à convecção devem aparecer enquanto o termo relativo à migração pode ser ignorado se for assumida a presença de eletrólito suporte.

O segundo termo do lado direito da equação (2) refere-se às 
reações químicas homogêneas presentes no mecanismo do processo estudado. Considere, por exemplo, o caso simples de um mecanismo de transferência quase reversível de um elétron precedido por um processo químico $(\mathrm{CE})$ :

$$
\begin{gathered}
P \rightleftharpoons O x \quad k_{1}, k_{-I} \\
O x+e^{-} \rightleftharpoons \operatorname{Red} \quad E^{0}, k^{0}, \alpha
\end{gathered}
$$

Um precursor $(\mathrm{P})$ dá origem à espécie oxidada $(O x)$, com constantes de velocidade $k_{I}$ e $k_{-I}$, que é reduzida à espécie Red com constante heterogênea $k^{0}$, potencial reversível $E^{0}$ e coeficiente de transferência $\alpha$. As três equações diferenciais (assumindo transporte por difusão e convecção), respectivamente para as concentrações das espécies $\mathrm{P}$ $(p)$, Ox $(o)$ e Red $(r)$, são:

$$
\begin{gathered}
\frac{\partial p}{\partial t}=\nabla \cdot\left(D_{p} \nabla p-\mathrm{u} c_{p}\right)-k_{I} p+k_{-I} o \\
\frac{\partial o}{\partial t}=\nabla \cdot\left(D_{o} \nabla o-\mathrm{u} c_{o}\right)+k_{I} p-k_{-I} o \\
\frac{\partial r}{\partial t}=\nabla \cdot\left(D_{r} \nabla r-\mathrm{u} c_{r}\right)
\end{gathered}
$$

Os ES são equipados com um compilador de reações químicas, um algoritmo originalmente proposto por Bieniasz, ${ }^{27}$ que traduz automaticamente um mecanismo químico escrito na forma de equações químicas como I e II em equações diferenciais como em 4a, 4b e 4c. No caso dos FES, os termos de transporte são automaticamente adicionados e o termo reacional deve ser introduzido pelo usuário.

A equação de Poisson só é utilizada quando uma distribuição não uniforme de potencial é esperada para uma determinada fase que compõe o sistema, ou seja, para meios que tenham condutividade relativamente baixa ou quando a corrente que circula é relativamente alta. Se o meio pode ser considerado eletricamente neutro, a densidade de carga $r$ é nula e a equação de Poisson se reduz à equação de Laplace.

As equações NPP são equações de continuidade e, portanto, assumem um meio isotrópico, contínuo e homogêneo. Se diferentes fases estão presentes (considere, por exemplo, celas eletroquímicas com diferentes compartimentos ou situações em que o transporte das espécies ocorre na solução eletrolítica e no material que recobre o eletrodo, como polímeros condutores ou filmes de óxidos) as equações devem ser escritas separadamente para cada meio com as respectivas constantes específicas para cada meio $\left(D_{i}, \mu_{i}, \varepsilon\right)$.

A utilização dos FES permite ainda realizar a chamada modelagem multifísica, isto é, outras equações além daquelas descritas nesta seção podem ser incluídas no modelo. Com a modelagem multifísica é possível abordar problemas envolvendo transporte de calor, campo magético, ${ }^{28}$ ondas sonoras ${ }^{29}$ e outros.

\section{Condições de contorno}

A solução das equações diferenciais parciais apresentadas requer um conjunto apropriado de condições de contorno. As condições de contorno estabelecem o valor da variável $\left(c_{i}, \phi\right)$ ou do seu gradiente $\left(\nabla c_{i}, \nabla \phi\right)$ em cada uma das fronteiras do domínio. No primeiro caso são denominadas condições de Dirichilet e no segundo caso são denominadas condições de Neumann. Para a equação de Nernst Planck as condições de Neumann equivalem ao fluxo de espécies através da fronteira e na direção normal a ela. Para a equação de Poisson as condições de Neumann equivalem ao campo elétrico na direção normal à fronteira. Condições mistas envolvendo tanto o valor da variável quanto o seu gradiente são denominadas condições de Robin.

No exemplo apresentado na Figura 1, apenas a equação de NernstPlanck está sendo considerada e existem 5 fronteiras denominadas $\delta \Omega_{1}$ a $\delta \Omega_{5}$ para as quais devem ser estabelecidas condições de contorno apropriadas. Nos FES isto pode ser feito simplesmente escolhendo a condição apropriada para cada fronteira de uma lista de opções. Assumindo que a altura do canal é muito maior que a camada de difusão pode-se considerar que a concentração da espécie eletroativa não é alterada em $\delta \Omega_{1}$, estabelecendo-se assim uma condição de Dirichilet para esta fronteira. A mesma condição é obtida para $\delta \Omega_{2}$ assumindo-se que o fluxo é suficiente para manter a concentração constante na entrada do canal. Para $\delta \Omega_{3}$ estabelece-se que o fluxo de espécies é nulo uma vez que as paredes do canal são impermeáveis. A fronteira $\delta \Omega_{5}$ corresponde à saída do canal para o descarte de solução. Nesta fronteira é estabelecido que a concentração externa é igual a zero. A fronteira $\delta \Omega_{4}$ corresponde ao eletrodo e nela o fluxo da espécie eletroativa é escrito em função da corrente faradaica que flui através do eletrodo. Se uma cinética de Butler-Volmer é assumida, o fluxo é uma função da própria concentração e do potencial do eletrodo, resultando em uma condição do tipo Robin.

\section{Cálculo de variáveis globais}

A solução das equações diferenciais do modelo fornece o valor das variáveis $c_{i}$ e $\phi$ em função do tempo e da posição. A partir destas variáveis outras grandezas espacialmente dependentes podem ser calculadas, tais como fluxos, campo elétrico e corrente elétrica. A estas variáveis que dependem da posição dá-se a denominação de variáveis distribuídas.

Em geral, estamos também interessados em calcular variáveis que são obtidas a partir da integração de uma variável distribuída em uma determinada região do espaço. Este tipo de variável, que depende unicamente do tempo, recebe o nome de variável global. Um exemplo típico de variável global é a densidade de corrente medida externamente $\left(i_{\text {ext }}\right)$ em um experimento de controle de potencial. A corrente externa é calculada a partir da integração da densidade de corrente local $(i=z F j)$ ao longo da superfície que define o eletrodo:

$$
i_{\text {ext }}(t)=z F l \oint_{\delta \Omega} j(s, t) d s
$$

na qual $s$ representa o vetor posição ao longo da superfície que define o eletrodo.

É importante ter em conta que a superfície $\delta \Omega$ que define o eletrodo pode ter dimensões menores do que a do eletrodo representado. Assim, a constante $l$ representa as demais dimensões não incluídas no modelo. No caso representado na Figura 1, $l$ representa a largura do canal. Em simulações unidimensionais, o eletrodo é representado por um ponto e $l$ representa a área do eletrodo e nas simulações tridimensionais $l$ é igual a 1 .

\section{MÉTODOS NUMÉRICOS}

Dentre os métodos numéricos existentes para solução de equações diferenciais parciais, os mais empregados em problemas de eletroquímica são o método das diferenças finitas e o método dos elementos finitos. Tem sido objeto de certa disputa na literatura qual destes dois métodos é mais adequado para os problemas eletroquímicos. Entretanto, é consenso que o método das diferenças finitas é mais intuitivo e de mais fácil aplicação, especialmente para problemas unidimensionais dependentes do tempo, enquanto o método dos elementos finitos é mais adequado para simulação de domínios irregulares. É importante mencionar que os FES aplicam, na realidade, uma combinação dos dois métodos para problemas dependentes do tempo: o método dos elementos finitos é aplicado às derivadas espaciais e o método das diferenças finitas é aplicado às derivadas no tempo. 
Os princípios básicos destes métodos bem como sua aplicação a problemas de eletroquímica estão bem descritos em diversas publicações ${ }^{30,31}$ e não serão tratados aqui. $\mathrm{O}$ ponto importante que desejamos tratar nesta seção refere-se à exatidão das soluções numéricas e sua relação direta com o esforço computacional exigido. Ambos os métodos aplicam uma malha de pontos (diferenças finitas) ou nós (elementos finitos) sobre o domínio do problema a partir da qual as equações diferenciais contínuas são transformadas em um sistema de equações algébricas discretas. Quanto mais densa a malha, maior a exatidão da aproximação numérica e maior o número de equações algébricas a ser resolvido. Como a solução destas equações algébricas é a etapa que demanda maior esforço computacional, aumentar a exatidão da solução através da diminuição da malha implica em maior tempo computacional para a solução do problema. Devido a este fato, para a maioria dos problemas eletroquímicos é computacionalmente ineficiente obter soluções com um grau aceitável de exatidão a partir do refinamento uniforme da malha sobre todo o domínio do problema. A estratégia que permite obter um compromisso ideal entre o tempo computacional e a exatidão da solução consiste em construir uma malha adaptada ao problema a ser solucionado, isto é, nas regiões onde ocorrem variações mais abruptas da variável problema, a malha é mais densa. Um exemplo de malha de elementos finitos aplicado ao problema apresentado na Figura 1 é mostrado na Figura 2. Nota-se que a malha é muito densa em torno do eletrodo onde a variação na concentração da espécie eletroativa é muito mais abrupta.

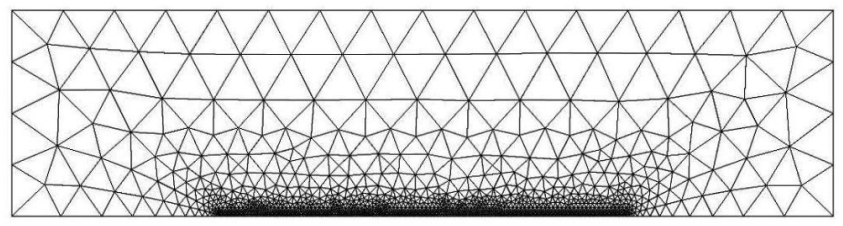

Figura 2. Malha de elementos finitos para a simulação de sistema microfluídico com microeletrodo de banda representado na Figura 1

Um considerável esforço de pesquisa tem sido feito visando o desenvolvimento de malhas adaptativas, ${ }^{15,32}$ isto é, algoritmos que capturam a variabilidade espacial da solução e refinam automaticamente a malha somente nos locais onde isto é necessário. Infelizmente, este tipo de algoritmo ainda não está operacional nos softwares comercialmente disponíveis, o que obriga o usuário a efetuar o ajuste da malha baseado no seu conhecimento do problema ou em simulações prévias. Nos ES a estratégia mais comumente utilizada é uma malha que se expande exponencialmente à medida que se afasta do eletrodo e um único parâmetro, que é o coeficiente de expansão da malha, é controlado pelo usuário. Nos FES um grande número de parâmetros permite o controle e refinamento da malha em diferentes regiões do domínio. Na malha apresentada na Figura 2 foram ajustados cinco parâmetros: tamanho máximo e tamanho mínimo dos elementos da malha global, tamanho máximo e tamanho mínimo dos elementos sobre o eletrodo e coeficiente de expansão da malha. O tempo necessário para a solução de um problema depende de diversos fatores como a complexidade do modelo, o refinamento da malha, o método utilizado para a solução das equações algébricas, a capacidade do processador e a memória disponível. De uma forma geral pode-se afirmar que em um computador com processador Intel i7 e 8 GB de memória, os problemas mais complexos podem exigir várias horas de processamento enquanto os problemas mais simples podem ser resolvidos em poucos segundos.

\section{EXEMPLOS DE APLICAÇÃO}

O principal foco nesta seção é mostrar através de alguns modelos típicos o tipo de informação que pode ser obtida a partir da eletroquímica computacional. Alguns trabalhos da literatura são mencionados sem a intenção de constituir uma revisão abrangente do tema. As soluções numéricas foram realizadas utilizando o software COMSOL Multiphysics 4.0a em um microcomputador com processador Intel i7 e 8 GB de memória.

\section{Estudos de mecanismos de reação}

A simulação computacional é uma ferramenta poderosa em estudos cinéticos de processos eletroquímicos. Neste tipo de estudo, a técnica experimental mais utilizada é a voltametria cíclica em eletrodos estacionários ou em condições hidrodinâmicas. Porém, se uma combinação complexa de picos sobrepostos é obtida experimentalmente, é praticamente impossível desvendar o mecanismo pela análise direta dos voltamogramas.

Um exemplo é mostrado na Figura 3, que apresenta simulações realizadas para um mecanismo $\mathrm{ECE}^{\#}$, no qual as espécies reduzidas de 2 pares redox $\left(a+e^{-} \rightleftharpoons b\right.$ e $\left.d+e^{-} \rightleftharpoons c\right)$ são, respectivamente, o reagente $(b)$ e o produto $(c)$ de uma reação química homogênea $(b \rightarrow c)$. Nestas simulações, equações similares à equação 4a para as 4 espécies envolvidas no mecanismo foram solucionadas sobre um domínio unidimensional.

Observam-se na Figura 3a voltamogramas simulados para diferentes valores das constantes de velocidade da reação química, para uma situação em que $E_{1}{ }^{0}<E_{2}{ }^{0}$. A análise quantitativa é dificultada pela ausência de uma relação analítica entre corrente e potencial e pela sobreposição dos picos. A dependência da intensidade relativa dos picos com a constante de velocidade está longe de ser trivial e não pode ser interpretada pela simples inspeção do voltamograma. No entanto, os resultados são facilmente racionalizados se considerarmos separadamente as contribuições dos dois processos eletroquímicos conforme observado na Figura 3b. Em particular, na região do primeiro pico, uma contribuição anódica correspondente à oxidação da espécie $c$, formada pela transformação química da espécie $b$ é subtraída da corrente de redução da espécie $a$. Assim, para constantes de velocidade químicas maiores, a formação de $c$ é maior, resultando na diminuição do primeiro pico de redução. No segundo pico observam-se claramente as contribuições sobrepostas dos dois processos de redução.

A partir da simulação, as diferentes proposições de mecanismos podem ser testadas e, fazendo o ajuste das curvas simuladas às experimentais, o valor das constantes de velocidade e outros parâmetros cinéticos e termodinâmicos podem ser obtidos. Uma excelente revisão abrangendo trabalhos com esta abordagem até 2002 foi publicada por Bieniasz. ${ }^{8}$ Diversos trabalhos podem ser encontrados na literatura mais recente. ${ }^{33-43} \mathrm{Um}$ texto abrangente, com foco na simulação de mecanismos de reação, foi publicado por Gosser. ${ }^{44}$

\section{Distribuição de corrente em célula de Hull}

Um dos aspectos chave para a aplicação prática de dispositivos eletroquímicos é a distribuição de corrente resultante da inter-relação entre os fenômenos interfaciais e os processos de transporte de massa e carga ao longo do sistema. Importantes características de dispositivos eletroquímicos tais como a densidade energética em baterias, capacitância específica em supercapacitores, propriedades

"Utilizamos aqui uma terminologia comum para descrever mecanismos eletroquímicos, na qual cada etapa de transferência interfacial de carga é indicada por um E ("electrochemical") e cada etapa química homogênea por um C ("chemical”). 


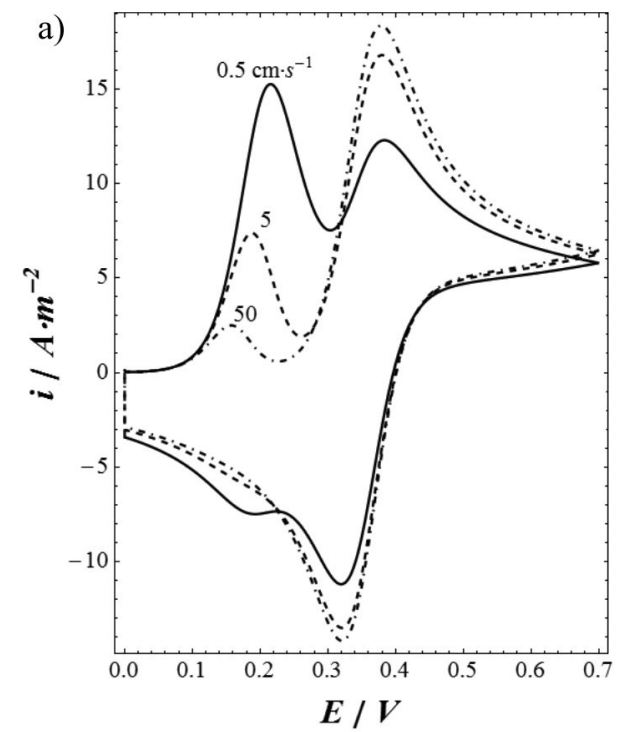

da densidade de corrente sobre o catodo. A Figura 4a mostra a distribuição de corrente no catodo, juntamente com a solução analítica aproximada, válida para o intervalo $0,186<z<0,941 .{ }^{45}$
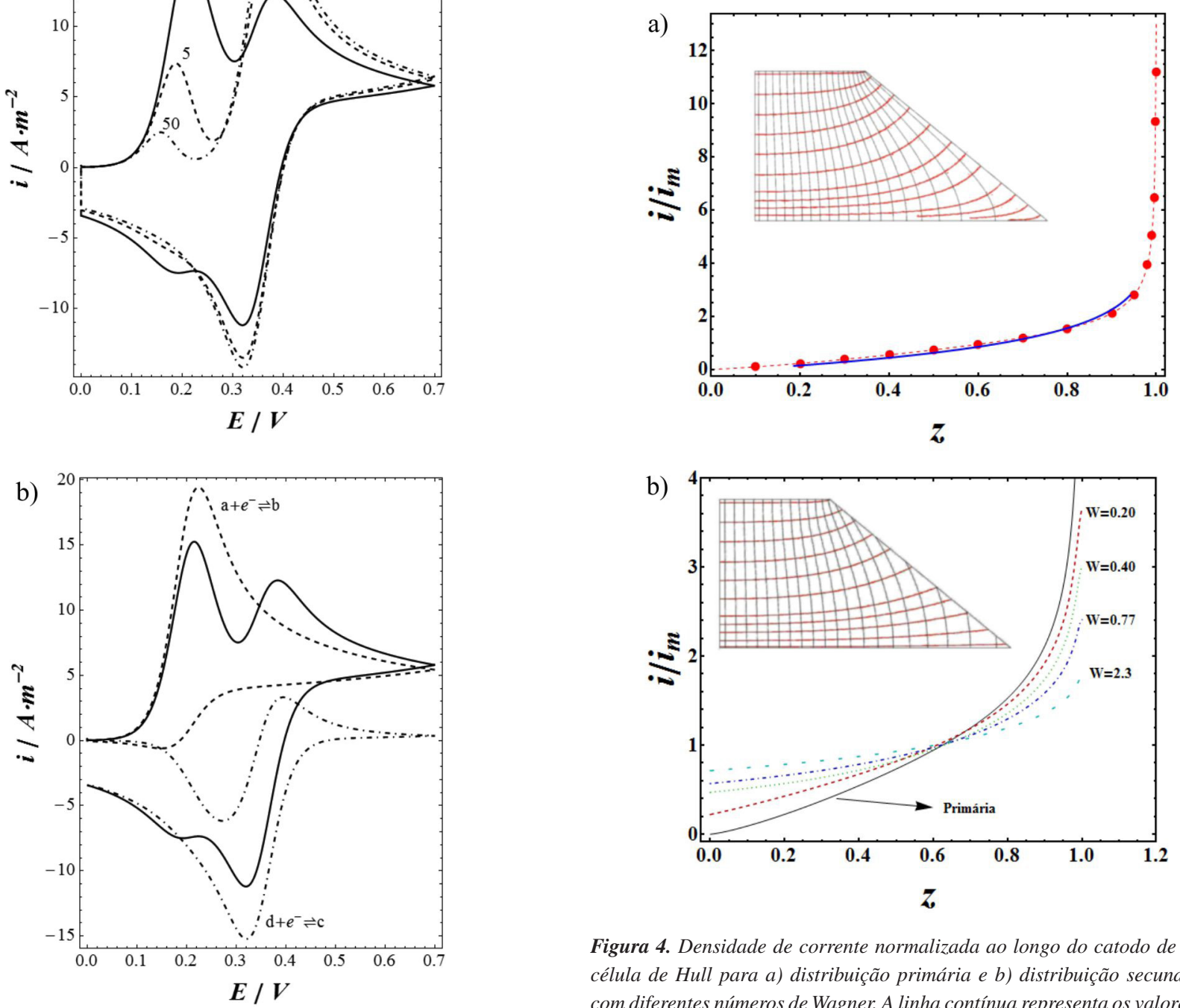

Figura 3. Voltametrias simuladas para um mecanismo ECE: a) Voltametrias simuladas para diferentes valores da constante de velocidade da reação homogênea, conforme indicado na Figura; b) Contribuição de cada um dos dois processos redox para a corrente total no caso em que a constante de velocidade do processo homogêneo é $0.5 \mathrm{~cm} \mathrm{~s}^{-1}$

estruturais e morfológicas de eletrodepósitos, eficiência de corrente em reatores eletroquímicos, para mencionar uns poucos exemplos, estão diretamente ligadas à distribuição de corrente. A modelagem computacional da distribuição de corrente em dispositivos eletroquímicos é importante não somente para um entendimento mais profundo do funcionamento do dispositivo bem como para fornecer uma base racional para projetos de engenharia e controle destes dispositivos.

Como um exemplo de modelagem da distribuição de corrente, apresentamos na Figura 4 distribuições calculadas de corrente e potencial em células de Hull. Quando os sobrepotenciais associados à reação eletródica e ao transporte de massa podem ser desconsiderados frente à resistência ôhmica do eletrólito, obtém-se uma distribuição de corrente primária, que depende unicamente da geometria da célula (inserto da Figura 4a). Neste caso, apenas a equação de Laplace é resolvida. Observa-se que as linhas de corrente que partem perpendiculares ao anodo curvam-se, tendendo a se concentrar na parte superior do catodo, mais próxima do anodo, resultando na variação

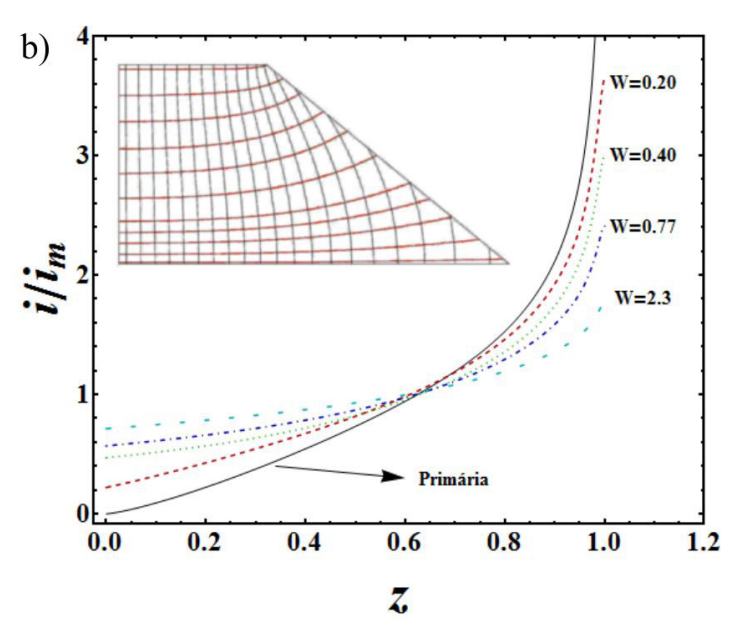

Figura 4. Densidade de corrente normalizada ao longo do catodo de uma célula de Hull para a) distribuição primária e b) distribuição secundária com diferentes números de Wagner. A linha contínua representa os valores de referência indicados na norma DIN 50957, válidos para $0.186<z<0.941$. Os insertos das figuras representam as curvas de contorno de potencial (verticais) e as linhas de corrente (horizontais) ao longo da célula de Hull

Se o sobrepotencial de transferência de carga é considerado, obtém-se uma distribuição secundária que depende da razão entre a resistência de transferência de carga e a resistência do eletrodo, expressa pelo número de Wagner (W). A distribuição secundária é obtida pela solução simultânea das equações de Nernst-Planck e de Laplace. A Figura 4b mostra distribuições secundárias de corrente sobre o catodo para diferentes números de Wagner. Para valores maiores de $\mathrm{W}$, a resistência de transferência de carga prevalece e a distribuição de corrente sobre o catodo é mais uniforme. $\mathrm{O}$ inserto da Figura mostra a distribuição de corrente e potencial ao longo da célula para $\mathrm{W}=0,77$ onde se observa que o encurvamento das linhas de corrente é muito menor comparado à distribuição primária.

O cálculo da distribuição de corrente tem sido aplicado ao estudo de diferentes sistemas eletroquímicos tais como baterias, ${ }^{46}$ células de

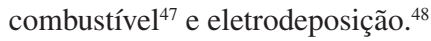

\section{Microscópio eletroquímico de sonda}

Uma das aplicações mais interessantes da eletroquímica computacional consiste na possibilidade de explorar sistemas em escala 
nanométrica. Como um exemplo, apresentamos a modelagem da microscopia eletroquímica de sonda, realizada a partir de um modelo originalmente proposto por Bard. ${ }^{49}$ A Figura 5 mostra a corrente de redução de uma espécie em solução em função da distância entre a sonda e o substrato, para sondas cônicas com diferentes razões de aspecto, considerando uma situação em que o substrato é condutor (Figura 5a) e uma situação em que o substrato é isolante (Figura 5b). Nos insertos estão representados a distribuição de concentração da espécie eletroativa ao longo do domínio do problema para a razão de aspecto $\mathrm{H}=1$. A cor azul (figuras coloridas na versão on-line) representa concentração nula e a cor vermelha (figuras coloridas na versão on-line) representa a concentração de bulk da espécie eletroativa. Note que um sistema de coordenadas cilíndricas foi utilizado de forma que o eixo horizontal representa a direção radial e o eixo vertical representa a direção axial. No inserto da Figura 5b estão indicados o raio da sonda (a), a altura da sonda (h), a distância da sonda até o substrato (d). A razão de aspecto é definida como $H=h / a$.

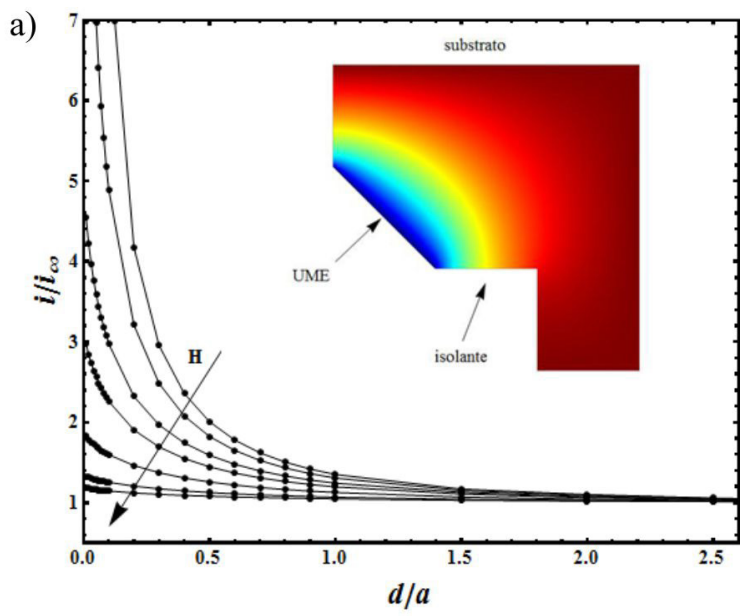

b)

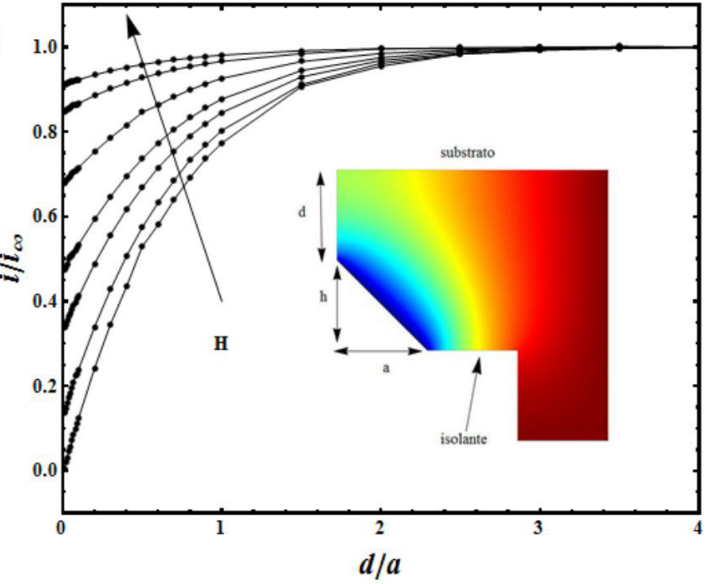

Figura 5. Efeito da razão de aspecto $(H)$ sobre a corrente medida na sonda para a) substrato condutor $e$ b) substrato isolante. Os insertos mostram a distribuição da concentração da espécie eletroativa ao redor da sonda

No caso do substrato condutor, a espécie reduzida que é produzida na sonda é oxidada novamente e um ciclo de retroalimentação positiva é formado. Note que a concentração na região entre a sonda e o substrato não se esgota, garantindo um fluxo de espécies eletroativas para a superfície da sonda. Como consequência, a corrente aumenta à medida que a sonda se aproxima do substrato. No caso em que 0 substrato é isolante, este impede o fluxo de espécies eletroativas até a sonda. Note a diminuição da concentração da espécie eletroativa na região entre a sonda e o substrato. Consequentemente, quanto menor a distância sonda-substrato, menor a corrente na sonda.
O efeito da razão de aspecto pode ser compreendido se considerarmos a direção predominante do fluxo das espécies eletroativas em cada caso. Deve-se considerar ainda que o aumento da razão de aspecto equivale a aumentar a inclinação do eletrodo em relação ao eixo horizontal. No caso em que o substrato é condutor, a direção predominante do fluxo por difusão é vertical e, logo, quanto mais inclinada está a superfície do eletrodo em relação ao eixo horizontal, menor o fluxo na direção normal ao eletrodo e, consequentemente, menor a corrente. No caso em que o substrato é não condutor, a direção predominante do fluxo por difusão é horizontal e, logo, o efeito contrário é observado.

\section{CONSIDERAÇÕES FINAIS}

Neste trabalho um roteiro básico para a utilização da eletroquímica computacional foi delineado, enfatizando que a existência de softwares comerciais permite a investigação de praticamente todos os problemas sem a necessidade de conhecimentos específicos de programação e matemática. Exemplos específicos de modelagem foram apresentados com o objetivo de apresentar o tipo de informação que pode ser obtida a partir da modelagem computacional e demonstrar o amplo espectro de problemas que pode ser tratado a partir desta técnica.

\section{AGRADECIMENTOS}

O autor agradece ao Prof. E. C. Pereira, que possibilitou o uso do programa COMSOL Multiphysics.

\section{REFERÊNCIAS}

1. Delahay, P.; J. Am. Chem. Soc. 1953, 75, 1190.

2. Nicholson, R. S.; Shain, I.; Anal. Chem. 1964, 36, 706.

3. Szabo, A.; Shoup, D.; J. Electroanal. Chem. 1982, 140, 237.

4. Cope, D. K.; J. Electroanal. Chem. 1997, 439, 7.

5. Mahon, P. J.; Oldham, K. B.; Electrochim. Acta 2004, 49, 5041.

6. Modern Aspects of Electrochemistry; Schlesinger, M., ed.; Springer New York: New York, 2009; Vol. 43.

7. Modern Aspects of Electrochemistry; Schlesinger, M., ed.; Springer New York: New York, 2009; Vol. 44.

8. Bieniasz, L. K.; Modern Aspects of Electrochemistry 2002, 35, 135.

9. Khaldeev, G. V; Petrov, S. N.; Russ. Chem. Rev. 1998, 67, 93.

10. Lopes, M. C.; Oliveira, C. P. de; Pereira, E. C.; Electrochim. Acta 2008, 53, 4359.

11. Pariona, M. M.; Müller, I. L.; Comput. Chem. 1998, 22, 377.

12. Feldberg, S. W. ; J.Electroanal. Chem. 1990, 290, 49.

13. Rudolph, M.; J. Electroanal. Chem. 2002, 529, 97.

14. Britz, D.; Østerby, O.; Strutwolf, J.; Electrochim. Acta 2012, 78, 365.

15. Gavaghan, D. J.; Gillow, K.; Süli, E.; Langmuir 2006, 22, 10666.

16. Bieniasz, L. K.; Electrochim. Acta 2011, 56, 6982.

17. http://www.basinc.com/products/ec/digisim/, acessada em Agosto 2013.

18. http://www.elchsoft.com/digielch/DigiElch7/Default.aspx, acessada em Agosto 2013.

19. http://www.cyfronet.krakow.pl/ nbbienia/elsim3ad.html, acessada em Agosto 2013.

20. http://www.comsol.com/products/multiphysics/, acessada em Agosto 2013.

21. http://www.pdesolutions.com/, acessada em Agosto 2013.

22. http://www.ansys.com/, acessada em Agosto 2013.

23. http://compton.chem.ox.ac.uk/john/Thesis/index.html, acessada em Agosto 2013.

24. Bard, A. J.; Faulkner, L. R.; Electrochemical methods: fundamentals and applications, 2nd ed., John Wiley \& Sons: New York, 2001. 
25. Koryta, J.; Dvorak, J.; Kavan, L.; Principles of Electrochemistry, $2^{\text {nd }}$ ed., John Wiley \& Sons: New York, 1987.

26. Newman, J.; Thomas-Alyea, K. E.; Electrochemical Systems, WileyInterscience: 2004.

27. Bieniasz, L. K.; Comput. Chem. 1996, 20, 403.

28. Sen, D.; Isaac, K. M.; Leventis, N.; Fritsch, I.; Int. J. Heat Mass Tran. 2011, 54, 5368 .

29. Henley, I. E.; Fisher, A. C.; Compton, R. G.; Banks, C. E.; J. Phys. Chem. B 2005, 109, 7843.

30. Britz, D.; Digital Simulation in Electrochemistry; $3^{\text {rd }}$ ed.; SpringerVerlag, 2005.

31. Nann, T.; Heinze, J.; Electrochem. Commun. 1999, 1, 289.

32. Harriman, K.; Gavaghan, D. J.; Süli, E.; J. Electroanal. Chem. 2004, $569,35$.

33. Burasov, A. V; Paddon, C. A.; Bhatti, F. L.; Donohoe, T. J.; Compton, R. G.; J. Phys. Org. Chem. 2007, 20, 144.

34. Cekirdek, P.; Solak, A. O.; Karakuş, M.; Aydin, A.; Yılmaz, H.; Electroanalysis 2006, 18, 2314.

35. Batchelor-McAuley, C.; Li, Q.; Dapin, S. M.; Compton, R. G.; J. Phys. Chem. B 2010, 114, 4094

36. Silvester, D. S.; Wain, A. J.; Aldous, L.; Hardacre, C.; Compton, R. G.; J. Electroanal. Chem. 2006, 596, 131.
37. Cekić-Lasković, I.; Marković, R.; Minić, D. M.; Volanschi, E.; J. Electroanal. Chem. 2011, 651, 50.

38. Karbarz, M.; Malyszko, J.; Electroanalysis 2008, 20, 1884.

39. Cekić-Lasković, I.; Minić, D. M.; Baranac-Stojanović, M.; Marković, R.; Volanschi, E.; Russ. J. Phys. Chem. A 2009, 83, 1571.

40. Salimi, A.; Amini, N.; Danyali, H.; Hallaj, R.; Electroanalysis 2006, 18, 1664.

41. Smith, E. T.; Davis, C. A.; Barber, M. J.; Anal. Biochem. 2003, 323, 114.

42. Streeter, I.; Wain, A. J.; Davis, J.; Compton, R. G.; J. Phys. Chem. B 2005, 109, 18500.

43. Núñez-Vergara, L. J.; Bontá, M.; Sturm, J. C.; Navarrete, P. A.; Bollo, S.; Squella, J. A.; J. Electroanal. Chem. 2001, 506, 48.

44. Gooser Jr, D. K.; Cyclic Voltammetry. Simulation and Analysis of Reaction Mechanisms, VCH Publishers: New York, 1994.

45. Norma 50950 DIN (Deutsches Institut für Normung). (1983).

46. Bernard, J.; Sciarretta, A.; Touzani, Y.; Sauvant-Moynot, V.; Oil Gas Sci. Technol. 2009, 65, 55.

47. Zhao, T. S.; Xu, C.; Chen, R.; Yang, W. W.; Prog. Energy Combust. Sci. 2009, 35, 275.

48. Mandin, P.; Cense, J. M.; Fabian, C.; Gbado, C.; Lincot, D.; Comput. Chem. Eng. 2007, 31, 980.

49. Zoski, C. G.; Liu, B.; Bard, A. J.; Anal. Chem. 2004, 76, 3646 